\title{
Five years' experience of PAS Port intravenous access system in adult cystic fibrosis
}

\author{
J. Burdon*, S.P. Conway*, P. Murchan**, M. Lansdown**, R.C.K. Kester**
}

Five years' experience of PAS Port intravenous access system in adult cystic fibrosis. J. Burdon, S.P. Conway, P. Murchan, M. Lansdown, R.C.K. Kester. OERS Journals Ltd 1998. ABSTRACT: An implantable venous access system provides a reliable and painless entry site for intravenous treatment. This study reports the authors' experience with such a system in adult patients with cystic fibrosis.

Sixty five $(87 \%)$ of 75 PAS Ports were placed successfully in 57 patients with cystic fibrosis. Because of early difficulties in advancing the catheter in patients whose veins had been traumatized by repeated courses of intravenous antibiotics, a technique was developed whereby venous entry was gained by direct subclavian puncture. This catheter insertion method was used in $53(82 \%)$ attempts and the catheter was then passed by subcutaneous tunnelling to the port site on the ventral aspect of the upper arm.

Fifty seven $(88 \%)$ insertions were successful under local anaesthetic. The major early and late complications were iatrogenic pneumothorax (six cases) and infection (five cases), respectively. Late complications were more common when there was coexisting disease, $e . g$. diabetes mellitus, or an acute severe respiratory exacerbation, or when the Port was used for parenteral feeding.

In conclusion, the PAS Port can be inserted safely by direct subclavian puncture. It was well tolerated and universally liked by the patients. Eur Respir J 1998; 12: 212-216.
*Regional Adult Cystic Fibrosis Unit, and ***Dept of Surgery, St James' and Seacroft University Hospitals, Leeds, UK.

Correspondence: S.P. Conway

Seacroft Hospital

York Road

Leeds

UK

Fax: 441132063540

Keywords: Cystic fibrosis intravenous access PAS Port

Received: December 301996 Accepted after revision March 51998
Patients with cystic fibrosis now have a mean lifespan of $40 \mathrm{yrs}$ [1]. The basis of management is daily physiotherapy, optimal nutrition and frequent antipseudomonal intravenous antibiotics. The value of the latter in the treatment of acute respiratory exacerbations is proven [2]. The Copenhagen group has shown further clear benefit from elective three monthly courses of intravenous antibiotics [3]. However, many older, severely affected patients require more frequent intravenous therapy. Some patients who are awaiting lung transplant receive continuous daily intravenous treatment. The potential for venous thrombosis, fibrosis and difficult access increases as more patients live into adulthood. Problems with venous access during treatment result in suboptimal therapy because of missed and late doses, and in discomfort and probably pain for the patient. Moreover, many patients are opting for homebased intravenous antibiotic treatment [4], which cannot be successful without secure venous access.

A totally implantable venous access system (TIVAS) provides a reliable and painless entry site for intravenous treatment. The earlier Port-A-Cath (Graseby Medical Ltd., Watford, UK) is effective but cosmetically unacceptable for the majority of adult patients. Since 1993 we have almost exclusively used the low-profile PAS Port (Graseby Medical Ltd.) (Fig. 1), which is half the length and onethird the height of the Port-A-Cath.

The PAS Port system was designed for the administration of chemotherapy. The manufacturer recommends forearm insertion under local anaesthetic into basilic or cephalic veins with catheter advancement into the right atrium. Correct positioning of the catheter tip is confirmed using the Cathfinder system, in which visual and auditory signals indicate that the cathfinder probe is directly over the tip of the catheter. There is no need for radiographical screening, although a chest radiograph should always be obtained after completion of the procedure. This method of insertion reduces the risk of iatrogenic pneumothorax almost to zero. However, patients with cystic fibrosis opting for TIVAS are naturally those whose veins have been traumatized by many courses of intravenous therapy. Almost by definition, their need for a TIVAS device suggests that intravascular advancement of the PAS Port catheter from antecubital fossa to right atrium will not be possible. Our early failure in advancing the catheter highlighted the need for a different catheter insertion technique in these patients.

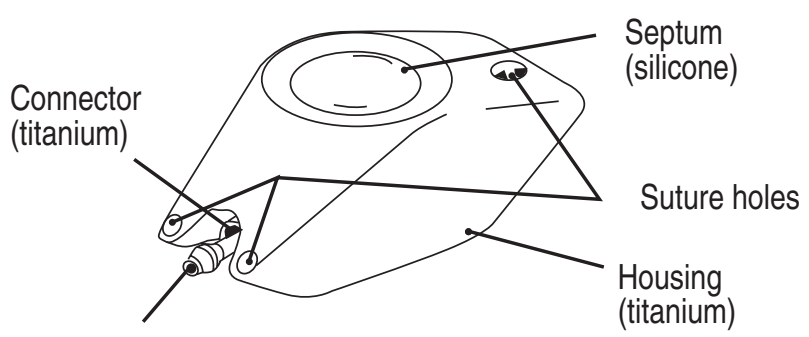

Outlet tube

(titanium)

Fig. 1. - PAS Port intravenous access device. 
Our five year experience of the PAS Port system was evaluated to assess the success rate of a novel insertion technique and to assess the reliability, complication rate and patient tolerance of this intravenous access device.

\section{Patients and methods}

The subclavian vein is cannulated under local anaesthetic, and the catheter inserted by the Seldinger technique and advanced to the correct position as determined by the PAS Port Cathfinder tracking system. Any areas of vascular fibrosis or thrombosis in the upper arm veins are bypassed. A transverse $1 \mathrm{~cm}$ skin incision is then made in the upper arm just medial to the insertion of the deltoid muscle, and a pocket made for the port (fig. 2). A tunnelling rod is passed subcutaneously to the subclavian incision and withdrawn with the catheter tip attached. The catheter is trimmed and attached to the port, which is then secured within its pocket.

Patients were observed overnight following PAS Port insertion. When not in use, the line was flushed monthly with Hep-Flush (6 mL, Leo Pharmaceutical Products, Ballerup, Denmark), using the specially angled needle so as not to damage the portal septum and a $10 \mathrm{~mL}$ or larger syringe so as not to apply too much pressure, always maintaining a positive pressure on the system once access was achieved. Patients were advised to avoid contact sports but otherwise to take no special precautions.

Criteria for offering patients the PAS Port TIVAS were not rigidly defined. Their use was discussed routinely with any patient in the unit whose home intravenous antibiotic course was aborted because of an inability to secure reliable venous access, and with any patient who repeatedly needed more than two intravenous lines during a twoweek course of intravenous antibiotic treatment. The consultant for cystic fibrosis or the clinical nurse specialist discussed the procedure for PAS Port insertion with all patients. All were shown the device and many accepted the offer to talk to another patient who had had a PAS Port placed.

Criteria for line infection, other than a positive blood culture, were any unexplained fever, failure of a fever to resolve with broad-spectrum intravenous antibiotic cover, or unexplained local discomfort.

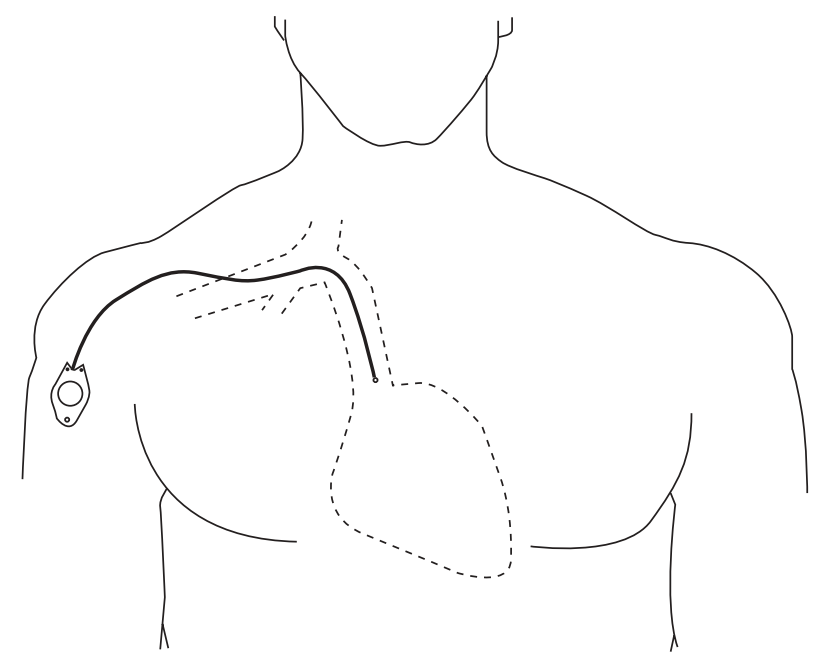

Fig. 2. - Modified insertion technique of PAS Port device.

\section{Results}

The results of 11 studies (this study; [5-14]) are summarized in table 1 . Sixty-five (87\%) of 75 PAS Port placements were successful at the first attempt in 57 patients. Six failures were due to an inability to cannulate the vein: once the subclavian, twice an antecubital fossa vein, and thrice the cephalic vein in the upper arm. Four attempts failed because of the use of local anaesthetic, twice each because of complaints of pain and fear of the procedure. In all, 10 failures occurred in eight patients, in seven of whom the PAS Port was subsequently successfully sited. The eighth received a Port-A-Cath.

Mean patient age was 21 yrs (range 12-48 yrs), mean forced expiratory volume in one second $\left(\mathrm{FEV}_{1}\right) 50 \%$ predicted normal (range 15-110), mean forced vital capacity (FVC) $70 \%$ predicted normal (range 29-143), and mean percentage weight for height $89 \%$ (range 70-120).

Of the 65 procedures that were successful at the first attempt, $57(88 \%)$ were performed under local anaesthetic, $53(82 \%)$ by direct subclavian puncture, $10(15 \%)$ by cephalic vein puncture and two $(3 \%)$ by basilic vein puncture.

Eight ports were replaced for the following reasons: Candida septicaemia (two ports), Pseudomonas aeruginosa infection in the line (one port), blocked line (one port) (Staphylococcus aureus was coincidentally isolated from the port after its removal), external trauma (one), leak from the port (one), pain during antibiotic infusion (one) and wound dehiscence (one).

Early complications numbered nine (14\%), i.e. pneumothorax (six), arterial puncture (two) and severe bruising (one). All pneumothoraces resolved without complication following standard chest drain insertion. Late complications numbered 12 (18\%), i.e. infection (Candida septicaemia (two), line infection with $P$. aeruginosa (one), port infection with $S$. aureus (one) and clinically suspected but unproven infection (one)), erosion of covering skin (three), leak from the port site (one), wound dehiscence (one), external trauma (one) and pain on antibiotic infusion (one).

The mean PAS Port duration in situ to date is 440 days (range 37-895), i.e. a total of 28,601 patient days. No patient has requested removal of the port. All patients who required removal of the port because of complications requested a replacement.

\section{Discussion}

PAS Port insertion by direct subclavian puncture as described above is an effective placement technique in adult patients with cystic fibrosis. The complication rate is acceptable. Suitably chosen patients tolerate the procedure well and enjoy the advantages of having a secure venous access system thereafter.

A subclavian approach for PAS Port insertion is needed in the majority of adults with cystic fibrosis. Most patients will have chest hyperinflation, increasing the risk of iatrogenic pneumothorax when this method is used. A postoperative chest radiograph is essential both to confirm the correct catheter position and to exclude any pneumothorax. Ideally, the procedure should be performed by a surgical team with ongoing experience of PAS Port placements to minimize the occurrence of pneumothorax and 


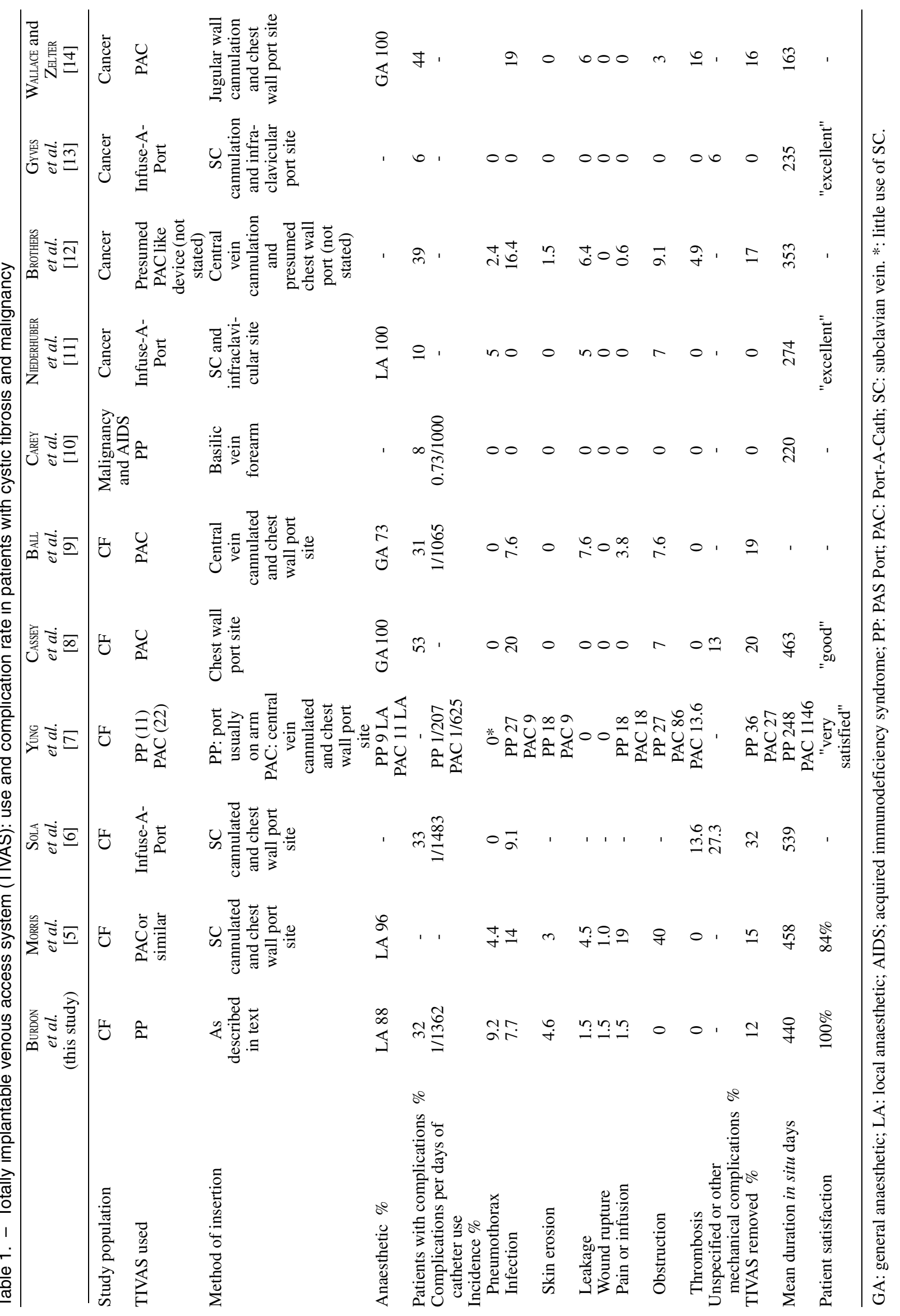


other technical complications. For instance, the one occurrence of wound dehiscence at the port site was due to the inadequate size of the pocket constructed for the port by a junior member of the surgical team. Although the majority of patients tolerate catheter placement under local anaesthetic, they must be prepared preoperatively to expect some intraoperative discomfort. The optimal procedure for children may still be the placement of a catheter under general anaesthetic. Two of the failures in the present series oc-curred because the patients were too frightened once in theatre. Both were young, aged 12 and 14 yrs, and had been referred from other hospitals. In retrospect, neither had been properly prepared for the procedure.

Coexisting illness may predispose patients to complications. Fungaemia must be suspected, looked for and promptly treated in any patient with a PAS Port in situ who develops an unexplained increase in body temperature [15]. Both patients with C. septicaemia in this series were already severely ill from cystic fibrosis-associated lung disease and further compromised by diabetes mellitus and pregnancy, respectively. Both were receiving parenteral nutrition through the Port, creating a milieu favourable to Candida growth. Erosion of the skin over the port site was seen in three patients, all of whom had diabetes mellitus which, in two cases, was poorly controlled owing to poor patient compliance.

No explanation was found for the patient who suffered pain on antibiotic infusion with the first port. Radiographic and Doppler studies showed no abnormality and symptoms did not recur with the placement port. Similar episodes of pain have, however, been reported in other series $[5,7,9,12]$. The incidence of overall complications in this series is higher than previously reported with PAS Port use for patients with malignancies [10], because catheterization of the subclavian vein is required and the catheters are required for the lifetime of the patient. When the PAS Port device was developed it was predicted that the incidence of thrombosis of the arm and subclavian vein would be greater than with ports placed on the chest wall [16]. Placing the port on the upper arm after subclavian puncture may reduce the potential risk to that of a port placed on the chest. In fact thrombotic complications appear to be particularly associated with a larger TIVAS [17-19]. None was noted with PAS Port use in this series or in the series by YuNg et al. [7], but a 4-14\% rate was reported for the Infuse-A-Port (Infusaid Corporation, Sharon, MA, USA) and Port-A-Cath in cystic fibrosis [6, 7, 9], and a 4-16\% rate in cancer patients [12, 14].

The overall complication rate, the complication rate per days of catheter use and the rate of catheter removal for complications in this series compare favourably with reports of the larger chest wall-sited Port-A-Cath and InfuseA-Port TIVAS in patients with cystic fibrosis [5-9] and malignancy or immunodeficiency [10-14]. Moreover, because of disease-related mortality, TIVAS used in patients with malignancy and acquired immunodeficiency syndrome (AIDS) had less time in situ to develop complications. This study also reported markedly fewer complications per day of catheter use than in the 11 PAS Ports inserted by the conventional route in patients with cystic fibrosis [7], i.e. 1 in 1,362 days compared with 1 in 207 days, and a reduced rate of catheter removal, i.e. $12 \%$ to $36 \%$. These differences may be explained by the different technique used for catheter insertion and port placement.
Implantable venous access catheters are popular with patients $[5,7-9,11,13,20,21]$. The technique described in this paper maintains the cosmetic advantages of the PAS Port. It is perhaps even better than forearm placement because the port is less obvious on the upper arm, where there is more subcutaneous fat, and where it is easily covered by clothes such as a T-shirt. In addition to the decreased disturbance of body image, the PAS Port TIVAS has advantages over the earlier Port-A-Cath system in that no radiology is required during the procedure and, therefore, there is no need to secure the services of a radiographer and neither patients nor staff are exposed to additional radiation. However, the authors experience leads to the suggestions that total parenteral nutrition should only be given through the port if essential, diabetes mellitus must be well controlled and all febrile episodes must be vigorously investigated.

In conclusion, the PAS Port intravenous access device can be inserted safely by cannulation of the subclavian vein, a technique which bypasses any upper arm vein thrombosis or fibrosis. The late complication rate compares favourably with that of ports inserted by the conventional route. Patients were generally very satisfied with the procedure and outcome.

\section{References}

1. Elborn JS, Shale SJ, Britton JR. Cystic fibrosis: current survival and population estimate to the year 200. Thorax 1991; 46: 881-885.

2. Regelmann WE, Warwick WJ, Elliott GR, Clawson CC. Reduction of sputum Pseudomonas aeruginosa density by antibiotics improves lung function in cystic fibrosis more than do bronchodilators and chest physiotherapy alone. Am Rev Respir Dis 1990; 141: 914-921.

3. Szaf M, Hoiby N, Flensborg EW. Frequent antibiotic therapy improves survival of cystic fibrosis patients with chronic Pseudomonas aeruginosa infection. Acta Pediatr Scand 1983; 72: 651-657.

4. Pond MN, Newport M, Joanes D, Conway SP. Home $v$ hospital intravenous antibiotic therapy in the treatment of young adults with cystic fibrosis. Eur Respir J 1994; 7: 1640-1644.

5. Morris JB, Occhinero ME, Gauderer MWL, Stern RC, Doershuk CF. Totally implantable vascular access devices in cystic fibrosis: a four year experience with fifty-eight patients. J Pediatr 1990; 117: 82-85.

6. Sola JE, Stone MM, Wise B, Colombaeni PM. Atypical thrombotic and septic complications of totally implantable venous access devices in patients with cystic fibrosis. Pediatr Pulmonol 1992; 14: 239-242.

7. Yung B, Campbell IA, Elborn JS, Harvey JS, Shale DJ. Total implantable venous access devices in adult patients with cystic fibrosis. Respir Med 1996; 90: 353-356.

8. Cassey J, Ford WDA, O'Brien L, Martin AJ. Total implantable system for venous access in children with cystic fibrosis. Clin Pediatr 1988; 27: 91-95.

9. Ball ABS, Duncan FR, Foster FJ, Davidson TI, Watkins $\mathrm{RM}$, Hodson ME. Long-term venous access using a totally implantable drug delivery system in patients with cystic fibrosis end bronchiectasis. Respir Med 1989; 83: 429-431.

10. Carey PC, Mann DV, Pearce SZ, et al. Long term circulatory access via a peripheral implantable port. Br J Surg 1993; 80: 600-601. 
11. Neiderhuber JE, Ensminger WD, Gyves JW, Leipman M, Doan K, Cozzi E. Total implanted venous and arterial access system to replace external catheters in cancer treatment. Surgery 1982; 92: 706-711.

12. Brothers TE, Von Moll LK, Niederhuber JE, Roberts JA, Walker-Andrews S, Ensminger WD. Experience with subcutaneous infusion ports in 300 patients. Gynecol Obstet 1988; 166: 295-301.

13. Gyves J, Ensminger WD, Neiderhuber JE, et al. Total implanted systems for intravenous chemotherapy in patients with cancer. Am J Med 1982; 73: 841-845.

14. Wallace J, Zeltzer PM. Benefits, complications, and care of implantable infusion devices in 31 children with cancer. J Pediatr Surg 1987; 22: 833-838.

15. Horn CK, Conway SP. Candidaemia: risk factors in patients with cystic fibrosis who have totally implantable venous access systems. J Infect 1993; 26: 127-132.

16. Starkhammer H, Bengtsson M, Morales O. Fibrin sleeve formation after long term brachial catheterisation with an implantable port device. Eur J Surg 1992; 158: 481484.

17. Peckham DG, Hill J, Manhire AR, Knox AJ. Resolution of superior vena cave obstruction following thrombolytic therapy in a patient with cystic fibrosis and a longer term indwelling catheter. Respir Med 1994; 88: 627-629.

18. Yung B, Eborn JS, Campbell IA, Summers Y, Beckles M, Woodcock AA. Thrombo embolism related to a Port-ACath device in a patient with cystic fibrosis. Thorax 1997; 52: 98-99.

19. Conway SP, Sivanathan UM. Superior vena cave obstruction in a patient with cystic fibrosis and a long term indwelling catheter. Respir Med 1995; 89: 396-397.

20. Lilienberg A, Bengtsson M, Starkhammer H. Implantable devices for venous access: nurses' and patients' evaluation of three different port systems. J Adv Nurs 1994; 19: 21-28.

21. Davies MJ, Wilson RG, Nixon SJ. Implantable venous access catheters: what the patients say. $J R$ Coll Surg Edinb 1992; 37: 125-126. 